\title{
Head Pre-Cooling Improves Symptoms of Heat-Sensitive Multiple Sclerosis Patients
}

\author{
Luke F. Reynolds, Christine A. Short, David A. Westwood, Stephen S. Cheung
}

\begin{abstract}
Background: Damage to the central nervous system by Multiple Sclerosis (MS) leads to multiple symptoms, including weakness, ambulatory dysfunction, visual disturbances and fatigue. Heat can exacerbate the symptoms of MS whereas cooling can provide symptomatic relief. Since the head and neck areas are particularly sensitive to cold and cooling interventions, we investigated the effects of cooling the head and neck for 60 minutes on the symptoms of MS. Methods: We used a double blinded, placebo controlled, cross-over study design to evaluate the effects of head and neck cooling on six heat-sensitive, stable, ambulatory females with MS (Extended Disability Status Scale 2.5-6.5). To isolate the effects of perceived versus physiological cooling, a sham cooling condition was incorporated, where subjects perceived the sensation of being cooled without any actual physiological cooling. Participants visited the clinic three times for 60 minutes of true, sham, or no cooling using a custom head and neck cooling hood, followed by evaluation of ambulation, visual acuity, and muscle strength. Rectal and skin temperature, heart rate, and thermal sensation were measured throughout cooling and testing. Results: Both the true and sham cooling elicited significant sensations of thermal cooling, but only the true cooling condition decreased core temperature by $0.37^{\circ} \mathrm{C}\left(36.97 \pm 0.21\right.$ to $\left.36.60 \pm 0.23^{\circ} \mathrm{C}\right)$. True cooling improved performance in the six minute walk test and the timed up-and-go test but not visual acuity or hand grip strength. Conclusions: Head and neck cooling may be an effective tool in increasing ambulatory capacity in individuals with MS and heat sensitivity.
\end{abstract}

RÉSUMÉ: Le prérefroidissement de la tête améliore les symptômes de la sensibilité à la chaleur chez les patients atteints de sclérose en plaques. Contexte : Le dommage au système nerveux central causé par la sclérose en plaques (SP) est responsable d'une multitude de symptômes dont de la faiblesse, une dysfonction ambulatoire, des troubles visuels et de la fatigue. La chaleur peut exacerber les symptômes de SP alors que le refroidissement peut en améliorer les symptômes. Comme la région de la tête et du cou sont particulièrement sensibles au froid et aux interventions refroidissantes, nous avons étudié les effets d'un refroidissement de 60 minutes de la tête et du cou sur les symptômes de la SP. Méthodes : Nous avons utilisé un plan d'étude à double insu, contrôlé par placebo et avec permutation, pour évaluer les effets d'un refroidissement de la tête et du cou chez six femmes atteintes de SP (EDSS 2,5 à 6,5) sensibles à la chaleur, capables de marcher et dont l'état était stable. Nous avons créé une situation de refroidissement factice pendant laquelle les sujets percevaient la sensation de refroidissement sans qu'il y ait de refroidissement physiologique réel afin d'isoler les effets d'un refroidissement perçu par rapport à un refroidissement réel. L'étude comportait 3 visites de 60 minutes à la clinique pendant lesquelles un refroidissement réel, factice ou aucun refroidissement n'était appliqué au moyen du port d'un capuchon refroidissant personnalisé de la tête et du cou, suivi par une évaluation de la marche, de l'acuité visuelle et de la force musculaire. La température rectale et cutanée, le rythme cardiaque et la sensation thermique étaient mesurés pendant toute la durée du refroidissement et de l'évaluation. Résultats : Le refroidissement réel et le refroidissement factice ont provoqué des sensations significatives de refroidissement, mais seul le refroidissement réel a diminué la température centrale de $0,37^{\circ} \mathrm{C}(36,97 \pm$ 0,21 à $\left.36,60 \pm 0,23^{\circ} \mathrm{C}\right)$. Le refroidissement réel a amélioré l'exécution du test de la marche de 6 minutes et du test minuté up-and-go, mais pas l'acuité visuelle ou la force de préhension de la main. Conclusions : Le refroidissement de la tête et du cou peut être un outil efficace pour augmenter la capacité ambulatoire chez les patients atteints de SP qui sont sensibles à la chaleur.

Can. J. Neurol. Sci. 2011; 38: 106-111

Exercise has proven to be an excellent intervention for managing the physical and psychological symptoms of Multiple Sclerosis $(\mathrm{MS})^{1-3}$. However, up to $80 \%$ of MS patients experience a worsening of their condition upon an increase in body temperature ${ }^{4}$, including visual function ${ }^{5}$, weakness, spasticity, bladder symptoms, and numbness ${ }^{6}$. Anecdotal reports from many patients with MS suggest that sensitivity to heat is one of the primary barriers to performing daily activities and participating in exercise and/or rehabilitation, and this sensitivity can lead physicians to advise patients to avoid exercise because metabolic heat production can increase core temperature and thus exacerbate symptoms ${ }^{3}$. In turn, this lower level of physical activity can lead to a further loss of fitness, muscle tone, and most importantly, a decrease in function and quality of life $\mathrm{e}^{7,8}$. A decrease in core temperature can improve some symptoms of $\mathrm{MS}^{9}$, fatigue, lower body muscle strength, and the ability to perform activities of daily living ${ }^{10}$. This has been demonstrated primarily using liquid conditioning garments, featuring a tight-

From the School of Health and Human Performance (LFR, DAW, SSC), Department of Physical Medicine and Rehabilitation (CAS), Dalhousie University, Queen Elizabeth II Health Sciences Centre, Halifax, Nova Scotia; Department of Physical Education and Kinesiology (SSC), Brock University, St. Catharines, Ontario, Canada.

Received April 12, 2010. Final Revisions Submitted July 7, 2010.

Correspondence to: Stephen Cheung, Department of Physical Education and Kinesiology, Brock University, 500 Glenridge Avenue, St. Catharines, Ontario, L2S 3A1, Canada. 
fitting garment around the head, torso, or the whole body, with a network of tubing that fits tightly against the skin and cooling through conduction of cold water ${ }^{7,8,11,12}$. Schwid et al ${ }^{12}$ found that cooling the head and torso for 1 or 30 days resulted in a small but significant increase in ambulatory function measured by the 25 foot walk test, and visual function improved as measured by Snellen charts. Conductive cooling of the hand in combination with a mild vacuum to maintain vasodilation was reported to increase voluntary walking duration ${ }^{13}$. Also, an evaporation-based cooling system was used to decrease the surface temperature of the thighs in individuals with MS; significant improvements were seen for lower body muscle strength, nine hole peg test performance, and the 25 feet walk test, whereas hand grip strength, posture, and spasticity were not improved $^{14}$.

Currently, a major limitation to the therapeutic use of cooling modalities remains the lack of portability, the need for a large power source, and the overall bulkiness of the units. The head and neck forms a relatively small $(\sim 10 \%)$ fraction of the total skin surface, but can be highly efficient for applying a cooling stimulus ${ }^{15,16}$. Therefore, an effective head and neck cooling device would require less power and a smaller pump compared to a conventional torso or whole-body cooling unit. Following 30 minutes of head and neck cooling in men, rectal temperature decreased by $0.26^{\circ} \mathrm{C}$ and $0.20^{\circ} \mathrm{C}$ in the winter and summer seasons, respectively; for females, the decreases were $0.23^{\circ} \mathrm{C}$ and $0.03^{\circ} \mathrm{C}$, respectively ${ }^{7}$. The reason for the seasonal variation in thermal response to cooling remains unclear, and unfortunately the only functional measurement taken was hand grip strength, which increased significantly with cooling.

Due to the paucity of research on the efficacy of head and neck cooling in the reduction of a variety of MS symptoms, the purpose of this study was to determine if 60 minutes of head and neck pre-cooling would improve ambulation, visual acuity, and muscle strength, in a sample of ambulatory patients with MS. To control for any placebo effects that may be associated with the sensation of being cooled, a sham cooling condition was included in the experimental design, where the perception of cooling was elicited without any significant changes in core temperature.

\section{MeTHODS}

\section{Participants}

This study was approved by the appropriate institutional review board and all six female participants provided their written consent before commencing the study. Having only female participants was not an initial goal or intent of the authors. Participants were first screened by a physician to ensure that they met inclusion criteria for the study, including having definite MS as defined by the McDonald Criteria and selfreporting heat sensitivity or a worsening of MS symptoms in warm environments. Exclusion criteria included using antihypertensive, vaso-active or diuretic drugs, participating in another clinical trial, pregnancy, suffering from another neural or muscular disease, or having suffered an exacerbation within the past month. Upon the first visit, a physiatrist performed an Extended Disability Status Scale ${ }^{17}$ (EDSS) assessment as well as reviewed their medical history to ensure that they were clinically stable (EDSS score would not likely change during the trial). The average age, height, and weight, were $41.3 \pm 7.3 \mathrm{y}, 164.4 \pm 5.5$ $\mathrm{cm}, 80.0 \pm 28.3 \mathrm{~kg}$, respectively. Extended Disability Status Scale scores ranged from 2.5 to 6.5 , and participants had been diagnosed with MS for 2-17 years. To avoid hormonal influences and natural variations in body temperature, all participants were tested during the follicular phase of their menstrual cycle and testing occurred at the same time of day for each participant. Participants were instructed to refrain from caffeine, smoking, and taking any vasoactive medications prior to the study. Exercise was avoided for 24 hours prior to testing, and eating was avoided for three hours prior to testing. Water was available ad libitum and the testing environment was set at normal room temperature $\left(22^{\circ} \mathrm{C}\right)$ and consistent humidity $(\sim 40 \%)$ for all trials.

\section{Protocol}

This study was a double-blind crossover design, with three conditions randomly presented in a counter-balanced fashion; true cooling, sham cooling, and no cooling. Upon arrival at the clinic, participants self-inserted a core temperature probe (MonA-Therm Core, Mallinkrodt Medical, St. Louis, USA) $15 \mathrm{~cm}$ beyond the anal sphincter. In the sham cooling condition, subjects perceived a cooling stimulus but no physiological change in core temperature was expected, or observed. Participants were blinded to the presence or purpose of a sham cooling, but were instead informed that two identical cooling trials would be performed; blinding was not possible for the no cooling condition due to the lack of a cooling stimulus. During the no cooling condition, participants completed the test battery after resting for 60 minutes. For the true and sham cooling conditions, participants rested for 20 minutes to acclimatize to the environment before the 60 minutes of cooling, then performed the test battery. The test battery performed on all three visits consisted of hand grip strength, three visual acuity tests, the six minute walk test, the 25 foot walk test and the timed up and go test. The same physiotherapist conducted all of the measurements and was blinded to the cooling condition participants received.

\section{Head Cooling Protocol}

Following the initial 20 minute rest period, participants donned a custom-made head and neck cooling device (Life Enhancement Technologies, Santa Clara, USA). The cooling device consisted of a neoprene outside shell covering an inflatable bladder that ensured a snug fit around the head and neck of the participant. The inside of the cooling hood is made of a conductive plastic material through which the cooling fluid circulates. Sixty minutes of cooling was selected as a practical and reasonable time for MS patients to allocate for cooling in their daily lives. The cooling unit was turned on in advance to ensure that the circulating fluid was cooled and circulating properly, and was set at $10^{\circ} \mathrm{C}$ for the entire 60 minute true cooling period. For the no cooling condition, the hood was worn for $60 \mathrm{~min}$, but the circulator was not turned on. During the sham cooling condition the circulating fluid was initially set at $10^{\circ} \mathrm{C}$ and then after five minutes was increased to $24^{\circ} \mathrm{C}$ to prevent any true change in core body temperature. Both at 30 and 55 minutes the temperature was briefly decreased to $10^{\circ} \mathrm{C}$ to induce a cold 
Table 1: Summary of core temperature and thermal sensation during the cooling protocols

\begin{tabular}{|c|c|c|c|c|}
\hline \multirow[b]{2}{*}{ Condition } & \multicolumn{4}{|c|}{ Rectal Temperature $\left({ }^{\circ} \mathrm{C}\right)$} \\
\hline & $\mathbf{0}$ min & $30 \mathrm{~min}$ & $60 \mathrm{~min}$ & $70 \mathrm{~min}$ \\
\hline No Cooling & $37.05(0.28)$ & $37.00(0.29)$ & $37.01(0.34)$ & $37.01(0.33)$ \\
\hline True Cooling & $36.97(0.21)$ & $36.92(0.16)$ & $36.66(0.20)^{*}$ & $36.60(0.23)^{*}$ \\
\hline \multirow{2}{*}{ Sham Cooling } & $37.04(0.27)$ & $36.98(0.25)$ & $36.92(0.29)$ & $36.98(0.29)$ \\
\hline & \multicolumn{4}{|c|}{ Thermal Sensation } \\
\hline No Cooling & $5.2(0.4)$ & $5.2(0.4)$ & $5.2(0.4)$ & $5.2(0.4)$ \\
\hline True Cooling & $4.8(0.4)$ & $3.2(0.8) \dagger \dagger$ & $3.2(1.0) \dagger$ & $4.7(0.5)$ \\
\hline Sham Cooling & $4.6(0.9)$ & $4.0(0.7)$ & $3.4(0.9) \dagger$ & $4.2(0.4)$ \\
\hline
\end{tabular}

Thermal sensation scale ranges from 0 (unbearably cold) through 5 (neutral) to 9 (unbearably hot). * significant difference from time 0 minutes; $\dagger$ significant difference from no cooling; $\dagger \dagger$ significant difference from both no cooling and sham cooling

chill sensation. Subjects remained resting for a further ten minutes without cooling prior to beginning the test battery.

\section{Subjective Thermal Responses}

Previously validated thermal sensation scale ranging from 0 (unbearably cold) to 9 (unbearably hot) with 5 as neutral was used to determine perceptual responses to cooling ${ }^{18}$. Participants were instructed on how to appropriately rate their perception of their thermal state during familiarization, and subjective data were measured every five minutes throughout the cooling protocol.

\section{Performance Measurements}

Contrast letter acuity has proven to be reliable and valid for measuring visual function with MS patients ${ }^{19}$, and low contrast Sloan Charts consisting of varying degrees of grey letters of progressively smaller sizes on a white background were used to test visual acuity. Participants read from $100 \%, 2.5 \%$ and $1.25 \%$ contrast charts while they sat $2 \mathrm{~m}$ from the charts; each eye was measured independently and the number of letters read correctly was recorded. Upper body muscle strength was assessed by a hand grip dynamometer ${ }^{20}$. With the dominant hand starting from shoulder height, participants squeezed the dynamometer as hard as possible two times, and the higher of the two attempts was included in the analysis.

In order to determine the effectiveness of cooling on locomotor function, a number of ambulatory tests were performed. Participants performed the timed up and go test which measures the time taken for a participant to stand up from a chair, walk three feet, turn around and be seated ${ }^{21}$; this test provides insight into functional mobility ${ }^{22}$. The 25 foot walk test was used to measure walking speed; participants walked 25 feet as fast as they could two times and the scores were averaged. The 25 foot walk test is validated and correlates well with overall mobility and leg function ${ }^{23}$. The final ambulatory measurement performed was the six-minute walk test, a self-paced test where participants walked as far as possible; this is associated with the ability to perform activities of daily living and symptomatic fatigue. $^{24}$

\section{Statistical Analysis}

Core temperature and thermal sensation were analyzed using two-way (condition $\mathrm{x}$ time) repeated-measures ANOVAs to distinguish differences of time at 0,30,60 and 70 minutes, for the 3 cooling conditions. Values were analyzed at 70 minutes (10 minutes after cooling ended) to determine the effects of postcooling afterdrop in core temperature, and also because this represents a realistic time for subjects to begin activities following cooling. In order to compare the functional effects of no cooling, sham cooling, and true cooling conditions, a oneway (condition) repeated-measures ANOVA was used to analyze measures of visual acuity, ambulation, and muscle strength. If any main effects of condition, time or an interaction between the two were observed, a simple effects analysis was performed in order to determine where differences occurred.

\section{Results}

Following 60 minutes of cooling and 10 minutes of rest, rectal temperature significantly decreased by $0.37^{\circ} \mathrm{C}(\mathrm{p}<0.01)$ during the cooling condition, while the no cooling and sham cooling conditions did not affect rectal temperature (Table 1). True and sham cooling produced a significant perceptual response in that patients scored lower on the thermal sensation scale compared to the no cooling condition. The true and sham cooling conditions produced a significantly colder thermal sensation after 60 min compared to the no cooling condition $(\mathrm{p}<0.05)$.

There was a main effect of condition on the six minute walk test $(p=0.036)$. A pairwise analysis revealed that patients walked a further distance in the true cooling condition compared to the no and sham cooling conditions. The timed up and go performance was significantly improved by both the true and sham cooling compared to the no cooling condition $(p=0.004)$. There was no overall effect of the cooling conditions on the 


\begin{tabular}{|c|c|c|c|}
\hline & True Cooling & Sham Cooling & No Cooling \\
\hline $\begin{array}{l}25 \text { foot walk test (seconds) } \\
\mathrm{F}=2.462 \& \mathrm{P}=0.13\end{array}$ & $5.80(1.54)$ & $5.82(1.54)$ & $6.10(1.61)$ \\
\hline $\begin{array}{l}\text { Timed up and Go (seconds) } \\
\mathrm{F}=11.21 \& \mathrm{P}=0.0036\end{array}$ & $11.53(4.63) \dagger$ & $12.03(5.23) \dagger$ & $12.96(5.34)$ \\
\hline $\begin{array}{l}\text { 6-minute Walk test (metres) } \\
\qquad \mathrm{F}=4.731 \& \mathrm{P}=0.036\end{array}$ & $459.1(116.5) \dagger \dagger$ & $437.7(112.5)$ & $414.3(96.4)$ \\
\hline $\begin{array}{l}\text { Hand Grip Strength }(\mathrm{N}) \\
\mathrm{F}=1.699 \& \mathrm{P}=0.232\end{array}$ & $25.6(6.4)$ & $24.6(6.4)$ & $23.5(5.1)$ \\
\hline $\begin{array}{l}100 \% \text { Contrast Right Eye } \\
\text { F } 1.489 \& \mathrm{P}=0.272\end{array}$ & $54.3(2.5)$ & $51.3(1.0)$ & $49.5(2.8)$ \\
\hline $\begin{array}{l}2.5 \% \text { Contrast Right Eye } \\
F=1.540 \& P=0.261\end{array}$ & $27.5(4.6)$ & $25.9(3.5)$ & $24.3(4.3)$ \\
\hline $\begin{array}{l}1.25 \% \text { Contrast Right Eye } \\
F=0.863 \& \mathrm{P}=0.451\end{array}$ & $22.0(4.4)$ & $20.0(2.6)$ & $19.5(3.6)$ \\
\hline $\begin{array}{l}100 \% \text { Contrast Left Eye } \\
F=1.320 \& P=0.310\end{array}$ & $53.8(2.7)$ & $50.7(3.1)$ & $54.0(2.4)$ \\
\hline $\begin{array}{l}2.5 \% \text { Contrast Left Eye } \\
\mathrm{F}=3.837 \& \mathrm{P}=0.058\end{array}$ & $30.3(6.8)$ & $24.2(5.2)$ & $22.0(5.4)$ \\
\hline $\begin{array}{c}1.25 \% \text { Contrast Left Eye } \\
\mathrm{F}=3.171 \& \mathrm{P}=0.086\end{array}$ & $24.7(5.1)$ & $22.0(4.8)$ & $18.3(4.5)$ \\
\hline
\end{tabular}

$\dagger$ significant difference from no cooling; $\dagger \dagger$ significant difference from both no cooling and sham cooling

timed 25 foot walk test $(\mathrm{p}=0.135)$. Anecdotally, participants did not report any unusual changes in their day-to-day mobility or functional capacity, so the true cooling appears to have only short-term efficacy related to the acute temperature decrease.

Hand grip strength was not affected by the cooling $(\mathrm{p}=0.232)$ and neither was the right eye visual acuity at the $100 \%, 2.5 \%$, $1.25 \%$ contrast levels with p-values of $0.272,0.261$ and 0.451 respectively. The cooling condition did not affect visual acuity in the left eye at the $100 \%$ contrast level with an overall p-value of 0.310 . The visual acuity at the $2.5 \%$ and $1.25 \%$ contrast levels were not significantly affected by the cooling condition $(\mathrm{p}=$ 0.058 and $p=0.086$ respectively) but $F$ values of 3.38 and 3.17 respectively suggest a trend of improvement after the cooling compared to no cooling (Table 2).

\section{DISCUSSION}

Previous studies using liquid immersion ${ }^{25}$, cold air ${ }^{26}$, cold water ingestion $^{27}$, or liquid conditioning garments ${ }^{11,12}$ as an intervention for symptoms associated with heat in MS individuals have reported improvements of various symptoms such as ambulation, muscle strength, balance, fatigue, pain, and visual acuity ${ }^{11,26}$. This study was designed to investigate the clinical application of cooling the head and neck on symptoms and functional capacity of heat-sensitive MS patients. A unique feature of this study is that it is the first to include a comprehensive assessment of ambulation, visual function, and upper body muscle strength. In contrast, most studies investigated the effects of cooling on only one aspect of function with one or two performance tests, making it difficult to determine the holistic efficacy of pre-cooling in alleviating symptoms and improving activity for everyday use. The main findings of this study are that, following 60 minutes of head and neck cooling and 10 minutes of rest, rectal temperature significantly decreased by $0.37^{\circ} \mathrm{C}$. Hand grip strength and visual function were not affected by head and neck cooling. However, true cooling of just the head and neck significantly improved the distance covered during the six minute walk test and improved performance during the timed up and go test compared to both no and sham cooling. Each of the six subjects were "responders" in that each improved their six minute walk distance, with an overall average of $11 \%$ greater distance covered. In contrast, pharmaceutical interventions such as oral fampridine (4aminopyridine) improved 25 -foot walk test times by $\sim 25 \%$ in $35 \%(78 / 224) \mathrm{MS}$ individuals ${ }^{28}$. Despite our small sample size, the consistent response and the lack of side effects from our cooling intervention suggests that it may be a useful complement or replacement for pharmaceutical symptomatic treatments of MS to enhance exercise capacity.

Hand grip strength, which gives insight into overall upper body strength, was reported to improve following 30 minutes of 
head and neck cooling with a similar device ${ }^{7}$, but in our study head and neck cooling failed to improve hand grip strength. Visual function is often impaired in MS individuals, with an inverse relationship between temperature and visual acuity $26,27,29$. In one well-designed study, visual acuity was measured by lowcontrast Sloan Charts and was found to improve after 60 minutes of torso cooling; interestingly, the effects of cooling were more pronounced at lower levels of contrast ${ }^{12}$. In the current study, head and neck cooling did not improve visual acuity at any contrast level although in the left eye a trend was seen towards improvements at the $2.5 \%$ and $1.25 \%$ contrast levels. The effects of cooling on visual acuity appear to be more pronounced at lower contrast levels as exhibited from the results of this study and the Schwid et $\mathrm{al}^{12}$ study. Core temperature decreased to a level comparable to other MS cooling studies that have observed symptomatic relief. Therefore, it is unlikely that an insufficient decrease in core temperature was the reason for the lack of improvement in visual tests, hand grip strength and the 25 foot walk test. It is possible that significance was not observed in this study due to the small sample size; at the 2.5 and $1.25 \%$ contrast levels for the left eye, F values were over 3 indicating that with more participants a significant effect would be observed.

The placebo effect has been noted to be a limitation in interpreting results from other MS cooling studies ${ }^{30}$. Sham cooling in previous studies involved either running no ${ }^{14}$ or warm ${ }^{12,31}$ fluid through the cooling garment, whereas we opted to run cold fluid periodically through the hood. Thus, our sham cooling resulted in the perception of cooling while no decrease in body temperature was observed. With the exception of the timed up and go test, no functional or physiological outcomes were affected by the sham cooling. In the timed up and go test, both the perception of cooling and actual physiological cooling improved performance. Research in non-MS individuals suggest that skin temperature alone or the perception of the external thermal environment can be a major contributor to voluntary exercise intensity, such as found in the six-minute walk test. Cooler skin temperatures, independent of any changes in core temperatures, were reported to increase the distance cycled during a 30 minute cycling time trial ${ }^{32}$, while exercise in a $35^{\circ} \mathrm{C}$ environment elicited a more rapid decrease in voluntary power output when riding at the same perceived exercise intensity compared to 15 and $25^{\circ} \mathrm{C}^{33}$. However, the altered perception of cooling alone in our protocol did not appear sufficient to elicit an improved voluntary and self-paced performance. This may be due to a relatively low sample size, or that the relatively inactive subjects in our study are not able to monitor and self-regulate their exercise intensity as well as highly-trained individuals. However, as MS is primarily a central nervous system disease, decreasing deep body temperature with the true cooling appears essential to elicit a systematic improvement in functional exercise capacity.

\section{Practical Application}

Exercise has been shown to be a positive intervention for managing symptoms of MS. Better performances on the 25 foot walk test or the six minute walk test have been reported to be associated with higher self reported levels of function in persons with MS. Paltamaa et al $^{34}$ studied 120 ambulatory individuals with MS and found that the faster times on the 25 foot walk test and greater distance on the six minute walk test were two factors that were predictive of higher reported independence with self care, mobility and domestic life activities in individuals with MS. Therefore we feel our findings represent not only statistically significant but clinically significant changes.

\section{ConClusions}

This study demonstrates that the head and neck is a very effective and efficient area to apply cooling. Therefore, heatintolerant patients could use this technique to lower body temperature prior to exercise, physiotherapy, or activities of daily living, thus potentially increasing fitness, daily function and independence. This application is simple and could be done pre-exercise and be used in conjunction with agents such as fampridine to improve walking and mobility even further through increased endurance in exercise. Another benefit of head and neck cooling was that participants did not report any severe thermal discomfort or other side effects, and no shivering was observed during any of the trials. A further research direction may be testing the potential for cooling during activity itself on improving exercise tolerance in heat-intolerant MS individuals. In turn, this would enable greater mobility for the user and widen the clinical application for cooling strategies.

\section{ACKNOWLEDGEMENTS}

The authors thank the study participants and the nurses and physiotherapists that helped throughout the study. The project was funded by a Faculty of Health Professions Research Development Grant (Cheung and Westwood) from Dalhousie University.

\section{REFERENCES}

1. Dalgas U, Stenager E, Ingemann-Hansen T. Multiple sclerosis and physical exercise: recommendations for the application of resistance-, endurance- and combined training. Mult Scler. 2008; 14(1):35-53.

2. Motl RW, Gosney JL. Effect of exercise training on quality of life in multiple sclerosis: a meta-analysis. Mult Scler. 2008;14(1): 129-35.

3. Heesen C, Romberg A, Gold S, et al. Physical exercise in multiple sclerosis: supportive care or a putative disease-modifying treatment. Expert Rev Neurother. 2006;6(3):347-55.

4. Simons DJ. A note of the effect of heat and of cold upon certain symptoms of multiple sclerosis. Bull Neurol Inst NY. 1937;6: 385-6.

5. Uhthoff W. Untersuchungen uber die bei der multiplen herdsklerose verkommenden auguestorungen. Arch Psychiatr Nervenkr. 1889;21:303-20.

6. Marino FE. Heat reactions in multiple sclerosis: an overlooked paradigm in the study of comparative fatigue. Int $\mathbf{J}$ Hyperthermia. 2009;25(1):34-40.

7. Ku YT, Montgomery LD, Wenzel KC, et al. Physiologic and thermal responses of male and female patients with multiple sclerosis to head and neck cooling. Am J Phys Med Rehabil. 1999;78(5):447-56.

8. Ku YT, Montgomery LD, Webbon BW. Hemodynamic and thermal responses to head and neck cooling in men and women. Am J Phys Med Rehabil. 1996;75(6):443-50.

9. Capello E, Gardella M, Leandri M, et al. Lowering body temperature with a cooling suit as symptomatic treatment for thermosensitive multiple sclerosis patients. Ital J Neurol Sci. 1995;16(8):533-9.

10. Kinnman J, Andersson T, Andersson G. Effect of cooling suit treatment in patients with multiple sclerosis evaluated by evoked potentials. Scand J Rehabil Med. 2000;32(1):16-9. 
11. Ku YT, Montgomery LD, Lee HC, et al. Physiologic and functional responses of MS patients to body cooling. Am J Phys Med Rehabil. 2000;79(5):427-34.

12. Schwid SR, Petrie MD, Murray R, et al. A randomized controlled study of the acute and chronic effects of cooling therapy for MS. Neurology. 2003;60(12):1955-60.

13. Grahn DA, Murray JV, Heller HC. Cooling via one hand improves physical performance in heat-sensitive individuals with multiple sclerosis: a preliminary study. BMC Neurol. 2008;8:14

14. Meyer-Heim A, Rothmaier M, Weder M, et al. Advanced lightweight cooling-garment technology: functional improvements in thermosensitive patients with multiple sclerosis. Mult Scler. 2007;13(2):232-7.

15. Shvartz E. Effect of a cooling hood on physiological responses to work in a hot environment. J Appl Physiol. 1970;29(1):36-9.

16. Nunneley SA, Maldonado RJ. Head and/or torso cooling during simulated cockpit heat stress. Aviat Space Environ Med. 1983; 54(6):496-9.

17. Kurtzke JF. Rating neurologic impairment in multiple sclerosis: an expanded disability status scale (EDSS). Neurology. 1983;33 (11): $1444-52$

18. Gagge AP, Stolwijk JA, Hardy JD. Comfort and thermal sensations and associated physiological responses at various ambient temperatures. Environ Res. 1967;1(1):1-20.

19. Baier ML, Cutter GR, Rudick RA, et al. Low-contrast letter acuity testing captures visual dysfunction in patients with multiple sclerosis. Neurology. 2005;64(6):992-5.

20. Bohannon RW. Hand-grip dynamometry provides a valid indication of upper extremity strength impairment in home care patients. J Hand Ther. 1998;11(4):258-60.

21. Schoppen T, Boonstra A, Groothoff JW, et al. The timed "up and go" test: reliability and validity in persons with unilateral lower limb amputation. Arch Phys Med Rehabil. 1999;80(7):825-8.

22. Cattaneo D, Regola A, Meotti M. Validity of six balance disorders scales in persons with multiple sclerosis. Disabil Rehabil. 2006; 28(12):789-95

23. Solari A, Radice D, Manneschi L, et al. The multiple sclerosis functional composite: different practice effects in the three test components. J Neurol Sci. 2005;228(1):71-4
24. Savci S, Inal-Ince D, Arikan H, et al. Six-minute walk distance as a measure of functional exercise capacity in multiple sclerosis. Disabil Rehabil. 2005;27(22):1365-71.

25. Watson CW. Effect of lowering of body temperature on the symptoms and signs of multiple sclerosis. N Engl J Med. 1959; 261:1253-9.

26. Hopper CL, Matthews CG, Cleeland CS. Symptom instability and thermoregulation in multiple sclerosis. Neurology. 1972;22(2): $142-8$.

27. Scherokman BJ, Selhorst JB, Waybright EA, et al. Improved optic nerve conduction with ingestion of ice water. Ann Neurol. 1985; 17(4):418-9.

28. Goodman AD, Brown TR, Krupp LB, et al. Sustained-release oral fampridine in multiple sclerosis: a randomised, double-blind, controlled trial. Lancet. 2009;373(9665):732-8

29. Geller M. Appearance of signs and symptoms of multiple sclerosis in response to cold. Mt Sinai J Med. 1974;41(1):127-30.

30. Syndulko K, Ke D, Ellison GW, et al. Comparative evaluations of neuroperformance and clinical outcome assessments in chronic progressive multiple sclerosis: I. reliability, validity and sensitivity to disease progression. multiple sclerosis study group. Mult Scler. 1996;2(3):142-56.

31. Beenakker EA, Oparina TI, Hartgring A, et al. Cooling garment treatment in MS: clinical improvement and decrease in leukocyte NO production. Neurology. 2001;57(5):892-4.

32. Kay D, Taaffe DR, Marino FE. Whole-body pre-cooling and heat storage during self-paced cycling performance in warm humid conditions. J Sports Sci. 1999;17(12):937-44.

33. Tucker R, Marle T, Lambert EV, et al. The rate of heat storage mediates an anticipatory reduction in exercise intensity during cycling at a fixed rating of perceived exertion. J Physiol. 2006; 574(Pt 3):905-15.

34. Paltamaa J, Sarasoja T, Leskinen E, et al. Measures of physical functioning predict self-reported performance in self-care, mobility, and domestic life in ambulatory persons with multiple sclerosis. Arch Phys Med Rehabil. 2007;88(12):1649-57. 